3-OH-K und 3-OH-A sind bei neutralem und bei alkalischem $\mathrm{pH}$ sehr instabil, so $\mathrm{da} ß$ bei einem $z$. B. durch Sulfonamidmedikation alkalisch reagierenden Harn eine Abnahme der Konzentration bereits in der Blase vorkommen kann. Sehr niedrige Harnwerte sind dadurch erklärbar (11).

Aus diesem Grunde ist auch die Bestimmung der sogenannten „endogenen 3-OH-A“ (Tab. 2) notwendig, denn diese kann in ihrer Konzentration wäh- rend der Inkubation mit Kynureninase ( 3 Stdn.) abgenommen haben. Eine kürzere Inkubationszeit für die Kynureninasereaktion ist unter unseren Bedingungen nicht möglich, da sonst ein vollständiger Umsatz des 3-OH-K nicht gewährleistet ist. Durch Verwendung aktiverer Enzympräparate könnte jedoch eine kürzere Inkubationszeit erreicht werden.

Wir danken der Wissenschaftlichen Forschungsstelle im Verband der Cigaretten-Industrie für die Unterstützung dieser Arbeit.

\title{
Literatur
}

1. Musajo, L., A. Spada und D. Coppint, J. biol. Chemistry 196, 185 (1952). - 2. Musajo, L. und C. A. Benassi, Adv. clin. Chem. 7, 63 (1964). - 3. Boyland, E., in: D. M. Wallace (ed.), Tumouts of the bladder. S. 83. E. \& S. Livingstone Ltd, Edinburg-London (1959). - 4. Schievelbein, H. und E. Buchfink, Clin. chim. Acta (Amsterdam) 18, 291 (1967). - 5. Bonner, D. M. und $\mathrm{C}_{\mathrm{H}}$. YANOFSKY, Proc. nat. Acad. Sci. USA 35, 576 (1949). - 6. Kuss, E., Hoppe-Seyler's Z. physiol. Chem. 345, 195 (1966). - 7. WeBER, F. und O. WIss, in: Hoppe-Seyler-Thierfelder (Hrsg).
Physiologisch- und pathologisch-chemische Analyse, 10. Aufl. Bd. VIjB, S. 806. Springer-Verlag, Berlin-Heidelberg-New York (1966). - 8. Wiss, O., Z. Naturforsch. 11b, 54 (1956). - 9. Weber, F. und O. Wiss, Hoppe-Seyler's Z. physiol. Chem. 304, 232 (1956). - 10. Wiss, O., H. Simmer und H. Peters, HoppeSeyler's Z. physiol. Chem. 304, 221 (1956). - 11. Pipkin, G. und J. U. Schlegel, Proc. Soc. exp. Biol. Med. 120, 592 (1965). 12. Benassi, C. A., F. M. Veronese und A. de Antoni: Clin. chim. Acta (Amsterdam) 17, 383 (1967).

\section{Die Verwendung von o-Nitrophenylbutyrat als Fehlerquelle bei der Messung der Reaktivierbarkeit alkylphosphatvergifteter Serumcholinesterase durch 2-PAM ${ }^{1}$ )}

\author{
Von M. Geldmacher - v. Mallinckrodt und I. Kaiser \\ Aus dem Institut für gericbtliche Medizin und Kriminalistik der Universität Erlangen-Nürnberg \\ (Direktor: Prof. Dr. Dr. E. Weinig)
}

(Eingegangen am 2. Februar 1968)

Das Vorgehen von MAIN, Miles und BraId zur Bestimmung der Se'rum-Cholinesteraseaktivität unter Verwendung von o-Nitrophenylbutyrat als Substrat ist nicht geeignet zur Messung der Reaktivierbarkeit alkylphosphatvergifteter Serumcholinesterase durch 2-PAM, da schon 2-PAM allein unter den Bedingungen der Reaktion das Substrat nichtkatalytisch unter Freisetzung von o-Nitrophenol spaltet.

The method by MAIN, MILES and BraId for determination of human serum cholinesterase activity with o-nitrophenylbutyrate as substrate is not suitable for measuring activity after reactivation of alcylphosphate-poisoned serum cholinesterase by 2-PAM, since 2-PAM alone - under the conditions of the reaction - splits the substrate non-catalytically and liberates o-nitrophenol.

Die Aktivität der Serumcholinesterase kann zur Diagnose bestimmter Krankheitsbilder und von Vergiftungen mit Cholinesteraseblockern wie z. B. E 605 herangezogen werden. Methodisch stehen eine große Anzahl verschiedener Verfahren zur Verfügung, die nicht alle Acetylcholin als Substrat verwenden (siehe z. B. Stumpf (1), Koelle (2).

MaIN, Mirles und Brarp (3) haben 1961 das o-Nitrophenylbutyrat als Substrat eingeführt. МсСомв, LAmotta und Wetstone (4) empfehlen dieses Substrat zur Erkennung atypischer Serumcholinesterasen. SzAsz (5) hat einen kritischen Vergleich zwischen Acetylthiocholin, Butyrylthiocholin und o-Nitrophenylbutyrat als Substrate zur Cholinesterase-Aktivitätsbestimmung im Serum durchgeführt.

\footnotetext{
1) Abletrrungen: 2-PAM = Pyridin-2-aldoxim-N-Methyljodid E $605=$ Diäthyl-p-Nitrophenylthiophosphat, DFP = Diisopropylfuorphosphat, TEPP $=$ Tetraäthylpyrophosphat.
}

Auf Grund des Vorkommens atypischer Serumcholinesterasen mit meist geringerer Aktivität (6), der starken intraindividuellen Unterschiede in der Aktivität der normalen Enzyme, des Einflusses von Krankheiten sowie der Möglichkeit einer Cholinesteraseblockierung nicht nur durch Alkylphosphate, sondern auch durch Carbamate und viele andere Medikamente haben Friedberg und SakaI (7) ein Verfahren zur in vitroReaktivierung alkylphosphatvergifteter Cholinesterasen mit 2-PAM ausgearbeitet. Sie empfehlen, mit einer beliebigen . Methode die Aktivität ohne und mit 2-PAMZusatz zu prüfen. Ergebe sich durch 2-PAM eine Aktivitätssteigerung, so liege mit Sicherheit eine Vergiftung mit einem organischen Phosphorsäureester vor.

FRIEDbERg und SAKaI (7) selbst verwendeten die manometrische Methode von AMMON unter Benutzung der Warburg-Apparatur, als Substrat diente Acetyl- 
cholin. SUCKER (8) hat die Möglichkeit der Reaktivierbarkeit alkylphosphatvergifteter Cholinesterase durch 2-PAM mit zwei weiteren Verfahren, nämlich der elektrometrischen Messung der pH-Wert-Änderung sowie der kolorimetrischen Bestimmung des unverbrauchten Substrates geprüft. Auch er arbeitete mit Acetylcholin.

$\mathrm{Da}$ die Bestimmung der Serumcholinesteraseaktivität mit o-Nitrophenylbutyrat nach MarN, Miles und BRAID (3) einfach durchführbar ist und das Ergebnis innerhalb einer halben Stunde vorliegt, untersuchten wir, ob dieses System auch zur Messung der Reaktivierung des alkylphosphatgehemmten Ferments' mit 2-PAM geeignet ist.

\section{Methodik und Ergebnisse}

Die Bestimmung der Serumcholinesteraseaktivität erfolgte nach Main, Miles und Braid (3).

\section{Reagenzien}

0,5 Mr Phosphatpuffer $\mathrm{pH}$ 7,6. Vor Gebrauch 1:10 mit Wasser verdünnen $(0,05 \mathrm{M})$.

0,5M methanol. o-Nitrophenylbutyrat-Stammlösung.

Butanol-Phosphatpuffer: $95 \mathrm{~m} l 0,05 \mathrm{M}$ Phosphatpuffer werden mit $5 \mathrm{~m} /$ Butanol gut geschüttelt.

o-Nitrophenylbutyrat-Pufferlösung: $10 \mathrm{~m} l$ Butanol-Phosphat-

Puffer $+0,03 \mathrm{~m} l$ o-Nitrophenylbutyrat-Stammlösung.

Diisopropylfluorphosphat (DFP)-Lösung: $0,025 \mathrm{M}$ in iso-Propylalkohol.

Fermentlösung: $0,1 \mathrm{~m} l$ Serum oder Plasma in $25 \mathrm{~m} l$ eisgekühltem Butanol-Phosphatpuffer; nur $30 \mathrm{Min}$. haltbar.

50proz. Äthanol.

\section{Arbeitsgang}

Die Reaktion kann in Reagenzgläsern durchgeführt werden:

\begin{tabular}{cc}
\hline $\begin{array}{c}\text { A } \\
\text { (Probe) }\end{array}$ & $\begin{array}{c}\mathrm{L} \\
\text { (Leerwert) }\end{array}$ \\
\hline in diese gibt man: & \\
$5 \mathrm{~m} /$ o-Nitrophenylbutyrat- \\
Puffer-Lösung & $5 \mathrm{~m} l$ o-Nitrophenylbutyrat- \\
Puffer-Lösung \\
(jeder Ansatz enthält damit & $7,5 \mu \mathrm{Mol}$ Substrat) \\
- & $0,05 \mathrm{~m} l$ DFP-Lösung \\
$1 \mathrm{~m} /$ Fermentlösung & $1 \mathrm{~m} /$ Fermentlösung
\end{tabular}

Beide Gläser werden im Wasserbad bei $25 \pm 0,1^{\circ}$ genau 30 Min. inkubiert. Sodann erfolgen sofort die folgenden Zusätze:
$0,05 \mathrm{~m} l \mathrm{DFP}-$ Lösung

50proz. Äthanol ad 10,0 50proz. Äthanol ad 10,0
Anschließend wird in 1 oder $2 \mathrm{~cm}$-Küvetten die Extinktion von A bei $414 \mathrm{~nm}$ gegen $\mathrm{L}$ als Leerwert gemessen.

MAIN, MrLes und BraId (3) bestimmen die Menge des in Freiheit gesetzten o-Nitrophenols spektrophotometrisch. $\mathrm{Da}$ der $\mathrm{pK}-$ Wert von o-Nitrophenol bei 7,1 liegt, überwiegt bei dem $\mathrm{pH}-$ Wert des Inkubationsansatzes von 7,6 die ionisierte Form, die ein Absorptionsmaximum von $414 \mathrm{~nm}$ aufweist. o-Nitrophenylbutyrat zeigt bei $\mathrm{pH}$ 7,6 praktisch keine Absorption bei $414 \mathrm{~nm}$. Die o-Nitrophenol-Konzentration erwies sich bis zu einem Extinktionswert von 1,5 als lineare Funktion der Extinktion.

FrIEDbERG und SAKAI (7) verwendeten zur Reaktivierung alkylphosphatvergifteter Cholinesterasen 2PAM in einer Konzentration von $10^{-3} \mathrm{M}$. Bei orien- tierenden Versuchen mit Aktivitätsbestimmung von durch Systoxsulfoxyd ${ }^{1}$ ). in vitro gehemmter Serumcholinesterase nach MAIN, MILES und BRAID (3) stellten wir fest, daß nach Hinzufügen von 2-PAM (wir verwendeten ein Präparat der Firma Bayer, Leverkusen, mit einem Schmelzpunkt von 215-2160) zu dem Analysenansatz teilweise sehr viel höhere Extinktionen gemessen wurden als für das betreffende Humanserum vor Vergiftung und Reaktivierung. Deshalb wurde zu $5 \mathrm{ml}$ o-Nitrophenylbutyrat-Pufferlösung nur 2-PAM in $1 \mathrm{ml}$ Wasser gegeben. Sofort nach Zugabe der 2-PAMLösung färbte sich der Reaktionsansatz gelb, ohne daß ein Serumzusatz erfolgt war. Das Absorptionsmaximum der Lösung lag bei $414 \mathrm{~nm}$. Die wäßrige 2-PAMLösung sowiie die o-Nitrophenylbutyrat-Pufferlösung allein waren praktisch ungefärbt. Diese Ergebnisse legten den Gedanken nahe, daß 2-PAM allein in der Lage ist, aus o-Nitrophenylbutyrat eine gelb gefärbte Verbindung, wahrscheinlich o-Nitrophenol, in Freiheit zu setzen.

\section{Qualitative Untersucbung des Reaktionsproduktes}

Die qualitative Untersuchung der durch 2-PAMZugabe gelb gefärbten Lösung von o-Nitrophenylbutyrat in Phosphatpuffer $\mathrm{pH} \mathrm{7,6} \mathrm{erfolgte} \mathrm{dünnschicht-}$ chromatographisch unter Verwendung von Kieselgel G (Merck) und Toluol als Laufmittel. Zur Detektion wurde mit $2 \mathrm{~N} \mathrm{NH}_{4} \mathrm{OH}$ oder $\mathrm{NaOH}$ besprüht, wobei sich o-Nitrophenol sofort stark gelb, der Ester zunächst schwach gelb, dann zunehmend stärker anfärbte.

Die $R_{F^{-}}$-Werte betrugen für

$$
\begin{array}{lr}
\text { o-Nitrophenylbutyrat } & 0,28 \\
\text { o-Nitrophenol } & 0,49
\end{array}
$$

Folgende Ansätze wurden hergestellt:

a) $5 \mathrm{~m} l$ o-Nitrophenylbutyrat-Pufferlösung $+1 \mathrm{~m} l$ Wasser

b) $5 \mathrm{ml}$ o-Nitrophenylbutyrat-Pufferlösung $+1 \mathrm{mg} \mathrm{PAM} \mathrm{in} 1 \mathrm{~m} l$ Wasser.

Die Ansätze wurden 30 Min. bei $25^{\circ}$ gehalten, sodann mit Salzsäure angesäuert und mit Äther extrahiert. Die mit wasserfreiem Natriumsulfat getrocknete Ätherphase wurde eingedampft und der Rückstand mit Äthanol aufgenommen. Aliquote Teile hiervon wurden dünnschichtchromatographisch untersucht.

Dabei ergab sich, daß in Ansatz a) praktisch nur oNitrophenylbutyrat neben einer Spur o-Nitrophenol vorlag. Ansatz b) hingegen enthielt neben erheblich weniger o-Nitrophenylbutyrat eine größere Menge an o-Nitrophenol. Damit spaltet 2-PAM unter den genannten Bedingungen o-Nitrophenylbutyrat unter Freisetzung von o-Nitrophenol.

\section{Zeitlicber Ablauf der Reaktion}

Ům den zeitlichen Verlauf der Reaktion festzulegen, wurden jeweils $5 \mathrm{ml}$ o-Nitrophenylbutyrat-Pufferlösung und $1 \mathrm{mg}$ 2-PAM

1) Die Kurzbezeichnung Systoxsulfoxyd wird hier gebraucht für O,O-Diäthyl-S-2-(äthylsulfinyl)-äthÿlthiophosphat, Sarin für Methylphosphonsäure-fluor-isopropylester, Tabun für Dimethylamido-cyan-phosphorsäure-äthylester, Phosdrin für O,O-DimethylO-(1-Carbamethoxy-1-propen-2-yl)-phosphat), Toxogonin für $1,1^{\prime}$ Oxydimethyl-bis-(4-Hydroxy-iminomethylpyridiniumhydroxyd). 
in $1 \mathrm{~m} /$ Wasser bei $25^{\circ}$ neben einem anstelle der PAM-Lösung nur Wasser enthaltenden Leerwert über verschiedene Zeiten inkubiert, sodann nach der Vorschrift von MAIN, Mires und BraID (3) mit Alkohol auf $10,0 \mathrm{~m} /$ aufgefüllt und spektrophotometrisch bei $414 \mathrm{~nm}$ untersucht.

$\mathrm{Zu}$ den verschiedenen Zeiten wurden die in Tabelle 1 aufgeführten Extinktionen (jeweils Mittelwerte aus vier Einzelbestimmungen) gemessen, die nach Abzug des Leerwertes praktisch alle den gleichen Zahlenwert aufweisen.

Tab. 1

Zeitlicher Verlauf der nichtenzymatischen Spaltung von o-Nitrophenylbutyrat bei $25^{\circ}$ in Phosphatpuffer $\mathrm{pH} \mathrm{7,6}$ in Gegenwart und ohne 2-PAM

\begin{tabular}{cccc}
\hline $\begin{array}{c}\text { Inkubations- } \\
\text { zeit } \\
\text { [Min.] }\end{array}$ & $\begin{array}{c}\text { Ansatz (B) } \\
\text { mit 2-PAM }\end{array}$ & $\begin{array}{c}\text { Extinktionen } \\
\text { Leerwert (C) } \\
\text { ohne 2-PAM }\end{array}$ & B-C \\
\hline 5 & 1,10 & 0,01 & 1,39 \\
10 & 1,25 & 0,01 & 1,24 \\
15 & 1,30 & $-1,30$ \\
20 & 1,30 & 0,01 & 1,30 \\
25 & 1,40 & 0,06 & 1,39 \\
30 & 1,40 & 0,08 & 1,34 \\
45 & 1,50 & 0,07 & 1,42 \\
60 & 1,45 & 0,09 & 1,38 \\
75 & 1,80 & 0,13 & 1,41 \\
105 & 1,80 & 0,30 & 1,67 \\
135 & 1,90 & 0,32 & 1,50 \\
165 & 1,80 & 0,31 & 1,58 \\
195 & & & 1,49 \\
\hline
\end{tabular}

Daraus ergibt sich, daß die Freisetzung von o-Nitrophenol durch die angewandte PAM-Menge sehr schnell erfolgt. Der Endzustand ist schon nach höchstens 5 Min. erreicht.

\section{Beziebung zwischen 2-PAM-Menge und abgespaltenem o-Nitrophenol}

In einer weiteren Versuchsreihe wurde der Einfluß der PAM-Menge auf die in Freiheit gesetzte Menge an o-Nitrophenol untersucht.

Hierzu wurde wie oben beschrieben vorgegangen, mit dem Unterschied, daß außer $1,0 \mathrm{mg}$ auch $0,8,0,6,0,4$ und $0,1 \mathrm{mg}$ PAM in $1 \mathrm{ml}$ Wasser dem o-Nitrophenylbutyrat-Puffergemisch zugesetzt wurden. Die Inkubationszeit bei $25^{\circ}$ betrug $30 \mathrm{Min}$.

Die bei den einzelnen Ansätzen gemessenen Extinktionen (Mittelwerte aus je 4 Einzelbestimmungen) sind in Tabelle 2 wiedergegeben. Die Umrechnung der Extinktionen in $\mathrm{mg}$. o-Nitrophenol erfolgte anhand einer entsprechenden Eichkuve.

Tab. 2

Abhängigkeit der abgespaltenen o-Nitrophenolmenge von der zuge setzten 2-PAM-Menge (Phosphatpuffer pH 7,6; $25^{\circ}$; Inkubationszei 30 Min.)

\begin{tabular}{cc}
\hline 2-PAM im Versuchsansatz & $\begin{array}{c}\text { abgespaltene Menge o-Nitro- } \\
\text { mg } \\
\text { phenol } \\
\text { mg }\end{array}$ \\
\hline 1,0 & 0,365 \\
0,8 & 0,290 \\
0,6 & 0,225 \\
0,4 & 0,160 \\
0,1 & 0,040 \\
\hline
\end{tabular}

Es ist zu erkennen, daß geringere PAM-Mengen auch geringere Mengen o-Nitrophenol in Freiheit setzen.

In weiteren Ansätzen wurde geprüft, ob bei geringeren PAM-Mengen die Zeit der Bebrütung bei $25^{\circ}$ einen
Einfluß auf die Abspaltung von o-Nitrophenol hat. Gemessen wurde jeweils die Extinktion nach 5, 10, 15, 20,30 und 60 Min. Bebrütungszeit. Es zeigte sich wie schon in den Versuchen mit $1 \mathrm{mg} 2-\mathrm{PAM}$, daß Bebrütungszeiten über 5 Min. keinen Einfluß auf das Versuchsergebnis haben.

Setzt man aus Tabelle 2 die im Ansatz enthaltene 2PAM-Menge und die jeweils abgespaltene Menge an o-Nitrophenol, beide umgerechnet in mMol, miteinander in Beziehung, so ergeben sich die aus Tabelle 3 abzulesenden Quotienten:

Tab. 3

Beziehung zwischen eingesetztem 2-PAM und abgespaltenem o-Nitrophenol

\begin{tabular}{|c|c|c|c|c|}
\hline \multicolumn{2}{|c|}{$\begin{array}{l}\text { Eingesetzte 2-PAM- } \\
\text { Menge }\end{array}$} & \multicolumn{2}{|c|}{$\begin{array}{l}\text { abgespaltene Menge } \\
\text { o-Nitrophenol }\end{array}$} & \multirow[t]{2}{*}{$D / E$} \\
\hline $\mathbf{m g}$ & $\mathrm{mMol}$ (D) & $\mathrm{mg}$ & mMol (E) & \\
\hline $\begin{array}{l}1,0 \\
0,8 \\
0,6 \\
0,4 \\
0,1\end{array}$ & $\begin{array}{l}0,0038 \\
0,003 \\
0,0023 \\
0,0015 \\
0,0004\end{array}$ & $\begin{array}{l}0,365 \\
0,290 \\
0,225 \\
0,160 \\
0,040\end{array}$ & $\begin{array}{l}0,00264 \\
0,00208 \\
0,00161 \\
0,00115 \\
0,00029\end{array}$ & $\begin{array}{l}1,47 \\
1,44 \\
1,44 \\
1,30 \\
1,38\end{array}$ \\
\hline
\end{tabular}

Aus der Tabelle ergibt sich, daß o-Nitrophenylbutyrat stets im Überschuß im Ansatz vorhanden war, und zwar 2-20fach. Weiter läßt sich ablesen, daß jede angewandte 2-PAM-Menge o-Nitrophenol im molaren Verhältnis 1,5:1 in Freiheit setzte.

\section{Diskussion}

2-PAM ist in der Lage, unter den von MaIN, MrLes und BraID (3) gewählten Bedingungen (Phosphatpuffer $\mathrm{pH} 7,6 ; 25^{\circ}$ ) o-Nitrophenylbutyrat unter Freisetzung von o-Nitrophenol zu spalten, und zwar im Verhältnis 1,5 Mol eingesetztes 2-PAM : $1 \mathrm{Mol}$ o-Nitrophenol.

Diese Reaktion ist kein völlig vereinzelt dastehendes Phänomen. Es ist bekannt, daß eine größere Anzahl von Organophosphaten durch stickstoffhaltige Verbindungen gespalten werden können. WAGNER-JAUREGG und HACKLEY (9) zeigten z. B., daß die Hydrolyse von Diisopropylfuorphoshat (DFP) und Diäthylfluorphosphat in Gegenwart von Imidazol, Histidin, Pyridin und anderen ähnlich gebauten Verbindungen schneller abläuft. Bei der Beschleunigung der Hydrolyse von DFP durch Histidin und Imidazol handelte es sich um eine echte Katalyse, da Histidin und Imidazol nicht aus dem Reaktionsansatz verschwanden. Die Autoren nahmen die Bildung einer intermediären Verbindung zwischen Dialkylfluorphosphat und den Katalysatoren an, die dann sekundär wieder zerfällt. JANDORF (10) hat die Spaltungsbeschleunigung insbesondere von Methylisopropylfuorphosphat (Sarin) in Gegenwart von Hydroxylamin untersucht. Sie verlief bei Raumtemperatur und $\mathrm{pH}$ 7,5 nicht katalytisch, sondern stöchiometrisch. WAGNER-JAUREGG (11) fand, $\mathrm{da} B$ die Freisetzung von Fluorwasserstoff aus Sarin auch durch verschiedene Hydroxamsäuren beschleunigt wird, ebenso stellten GreEN und SAville (12) fest, $\mathrm{da} \beta$ in neutraler oder schwach alkalischer Lösung Oxime schnell mit Sarin reagieren und für jedes ge- 
spaltene Sarinmolekül ein Mol Oxim verbraucht wird. Green, Sainsbury, Saville und Stansfield (13) beobachteten eine Beschleunigung der Spaltung nicht nur von DFP und Sarin, sondern auch von TEPP und Tabun durch verschiedene stickstoffhaltige Verbindungen, insbesondere Hydroxamsäuren. GATTERDAM, Casida und Stoutamire (14) stellten fest, daß die Hydrolyse von Phosdrin bei $\mathrm{pH} 9$ etwa 3 mal schneller verläuft, wenn Hydroxylamin oder verschiedene PAMDerivate zugegen sind.

Quantitative Untersuchungen über die Abspaltung von Fluorwasserstoff aus DFP, u. a. durch Benzohydroxamsäure, Nicotinhydroxamsäure und Picolinhydroxamsäure stammen von Hackley, Plapinger, Stolberg und WAGNER-JAUREgG (15). Sie zeigten, daß pro Mol freigesetztem Fluorwasserstoff 1,5-2 Mole der Hydroxamsäuren aus dem Reaktionsansatz verschwanden. Dies stellte klar, daß die Hydroxamsäuren nicht als wahre Katalysatoren wirken, sondern selbst verändert werden. Dabei entsteht O-Phenyl-carbamyl-benzohydroxamat, $\mathrm{C}_{6} \mathrm{H}_{5}-\mathrm{CONHOCONH}-\mathrm{C}_{6} \mathrm{H}_{5}$, das isoliert und identifiziert werden konnte.

Bei der Reaktion von Sarin mit p-Methylbenzo-, pNitrobenzo-, p-Cyanobenzo-, Picolin- und Nicotinhydroxamsäuren wurden analoge Verbindungen erhalten.

Die genannten Organophosphatverbindungen können als einfache oder gemischte Säureanhydride aufgefaßt werden. Viele der aufgeführten stickstoffhaltigen Verbindungen, insbesondere die Oxime reagieren auch mit anderen Säureanhydriden wie $z$. B. Sulfonyl-HalogenVerbindungen und Essigsäureanhydrid. Schon WERNER und Piguet (16) hatten z. B. gefunden, daß eine alkalische Lösung von Benzilmonoxim mit Benzolsulfonsäurechlorid unter Spaltung des Säurechlorids reagiert.

HURD und BAUER (17) untersuchten die Spaltung von p-Toluolsulfonylchlorid mitBenzhydroxamsäure. Hierbei entsteht auch nach Hackley, Plapinger, Stolberg und WAGNER-JAUREGG (15) gleichfalls O-Phenylcarbamylbenzohydroxamat, so daß letztere den gleichen Reaktionsmechanismus wie bei der Spaltung von DFP annehmen.

GREEN und SAVILLE (12) prüften die Reaktion zwischen Essigsäureanhydrid und Hydroxyiminoaceton in praktisch neutraler Lösung, die fast augenblicklich zur Freisetzung von einem Mol Essigsäure führt. Anschließend kommt es dann zu einer langsameren $\mathrm{Ab}$ spaltung von Essigsäure aus dem gebildeten Oximacetat, wobei zwei weitere Mole Essigsäure und Blausäure entstehen. Der letztere Schritt. läuft mit zunehmendem $\mathrm{pH}$ schneller ab.

Nach unseren Ergebnissen kann o-Nitrophenylbutyrat als gemischtes Säureanhydrid aufgefaßt werden, das durch 2-PAM in einer nichtkatalytischen Reaktion gespalten wird. Der Verbrauch von etwa 1,5 Mol 2-PAM für jedes in Freiheit gesetzte Mol o-Nitrophenol läßt an einen ähnlichen Reaktionsablauf denken, wie er von HACKLEY und Mitarbeitern (15) formuliert wurde. Aufgrund dieser Reaktion ist die Methode von MaIN, MrLES und BraId (3) für den Nachweis einer Reaktivierung alkylphosphatgehemmter Serumcholinesterase durch 2-PAM nicht brauchbar.

Ähnliche Verhältnisse sind bei allen Substraten zu erwarten, die keine echten Ester, sondern wie o-Nitrophenylbutyrat Säureanhydride sind, also z. B. Phenylbenzoat (1), Naphthylacetat und Indoxylacetat oder Acetylsalicylsäure (2).

Orientierende Versuche haben ergeben, daß eine zu o-Nitrophenol führende Spaltung von o-Nitrophenylbutyrat auch mit Toxogonin eintritt.

Ein störungsfreier Verlauf ist zu erwarten, wenn Cholinester, insbesondere Acetylcholin Verwendung finden. Systematische Untersuchungen zu dieser Frage sind im Gange.

Die Arbeit wurde mit Hilfe der Deutschen Forschungsgemeinschaft durchgeführt, wofür wir auch an dieser Stelle unseren besten Dank aussprechen.

\section{Literatur}

1. Stumpf, Ch., Z. Vitamin-, Hormon u. Fermentforsch. (Wien) 8, 36 (1956). - 2. Koelle, G. B., Cholinesterase and anticholinesterase agents. Ergänzungswerk Band XV des Handbuchs der Experimentellen Pharmakologie. Hrsg. O. EIçHLER und A. FARAk. Springer, Berlin-Göttingen-Heidelberg (1963).-3. MaIN, A. R., K. E. Mrles und P. E. Brard, Biochem. J. 78, 769 (1961). 4. Mc Сомв, R. B., R. V. LA Motra und H. J. Wetstone, Clin. Chem. (New York) 11, 645 (1965). - 5. SzAsz, G., HoppeSeyler's Z. Physiol. Chem. 348, 1246 (1967). - 6. GoedDE, H.W., A. Doenicke und K. Altrand, Pseudocholinesterasen Springet, Berlin-Heidelberg-New York (1967). - 7. Friedberg, K. D. und F. SAKAI, Dtsch. Zschr. gerichtl. Med. 47, 580 (1958). 8. SuCKER, H., Enzymatische Methoden. in: GadAmERs Lehrbuch der chemischen Toxikologie und Anleitung zur Ausmittelung der
Gifte, Bd. II. Hrsg. E. Graf und Fr. R. Preuss. Van den Hoek und Rupprecht, Göttingen (1966). - 9. WAGNER-JAUREGG, T. und B. E. Hackley jr., J. Amer. chem. Soc. 75, 2125 (1953). - 10. JANDORF, B., J. Amer. chem. Soc. 78, 3686 (1956). - 11. WAGNERJAUREgG, T., Arzneimittel-Forschung Aulendorf 6, 194 (1956). 12. Green, A. L. und B. Saville, J. Chem. Soc. (London) 1956, 3887. - 13. Green, A. L., G. L. Sainsbury, B. Saville und M. Stansfield, J. Chem. Soc. (London) 1958jII, 1583. - 14. Gatrerdam, P. E., J. E. Casida und D. W. Stoutamire, J. econ. Ent. 52, 270 (1959). - 15. Hackley, B. E. jr., R. Plapinger, M. Stolberg und T. Wagner-Jauregg, J. Amer. chem. Soc. 77, 3651 (1955). - 16. Werner, A. und A. Piguer, Ber. dtsch. chem. Ges. 37, 4295 (1903). - 17. HuRd, Сh. D. und L. BAUER, J. Amer. chem. Soc. 76, 2791 (1954).

Priv.-Doz. Dr. Dr. M. Geldmacher-v. Mallinckrodt 852 Erlangen,

Universitätsstr. 22 